\title{
Invasive species management in two-patch environments: Agricultural damage control in the raccoon (Procyon lotor) problem, Hokkaido, Japan
}

\author{
Koji Kotani* ${ }^{*}$ Hiromasa Ishii ${ }^{\dagger}$ Hiroyuki Matsuda $^{a}$ Tohru Ikeda
}

December 21, 2008

\begin{abstract}
We develop discrete-time models for analyzing the long run equilibrium outcomes on invasive species management in two-patch environments with migration. In particular, the focus is upon a situation where removal operations for invasive species are implemented only in one patch (controlled patch). The new features of the model are that (i) asymmetry in density dependent migration is considered, which may originate from impact of harvesting as well as heterogeneous habitat conditions, and (ii) the effect of density-dependent catchability is well-taken to account for the nature that the required effort level to remove one individual may rise as the existing population decreases. The model is applied for agricultural damage control in the raccoon problem that has occurred in Hokkaido, Japan. Numerical illustrations demonstrate that the long run equilibrium outcomes highly depend on the degree of asymmetry in migration as well as the sensitivity of catchability in response to a change in the population size of invasive species. Furthermore, we characterize the conditions under which the economically optimal effort levels are qualitatively affected by the above two factors and aiming at local extermination of invasive species in the controlled patch is justified.
\end{abstract}

Keywords Catchability $\cdot$ Density dependent migration $\cdot$ Local extermination $\cdot$ Metapopulation $\cdot$ Removal effort

\footnotetext{
${ }^{*}$ Corresponding author: e-mail: kkotani@iuj.ac.jp. Assistant Professor, International Development Program, Graduate School of International Relations, International University of Japan, 777 Kokusaicho, Minami-Uonuma, Niigata 949-7277, Japan

${ }^{\dagger a}$ Faculty of Environment and Information Sciences, Yokohama National University, 79-7 Tokiwadai, Hodogaya-ku, Yokohama, Kanagawa 240-8501, Japan

${ }^{\ddagger}$ Graduate School of Letters, Hokkaido University, Kita 10 Nishi 7, Kita-ku, Sapporo Hokkaido, 060-0810, Japan
} 


\section{Introduction}

"The problem of invasive species and their control is one of the most pressing applied issues in ecology today (Hastings et al 2006)."

The invasive species have increasingly been acknowledged as a global threat, since they could fundamentally destroy indigenous ecosystem after its establishment (Shigesada and Kawasaki 1997; Perrings et al 2000a). Although there are several unique characteristics accompanied with invasive species that contribute to social damage, one critical feature is that they tend to spread or disperse very quickly after they succeed in invasion. Such quick dispersion partly reflects the fact that native species do not possess defensive skills against newcomers in many cases (Perrings et al 2000b).

Many governmental attempts have been made to eradicate the established invasive species. Unfortunately, however, only a few succeeded, and most of them failed especially when the habitat is sufficiently large (Bomford and O'Brien 1995). That is, the management official ends up halting eradication attempts (Bomford and O'Brien 1995; Myers et al 1998; Clout and Veitch 2002). When the invasive species are widespread in a large habitat, catchability could decrease in response to a decline in the population size of invasive species. This implies that the cost of removing the last 1-10\% population becomes prohibitively expensive, and thus achieving extermination appears to be extremely difficult (Bomford and O'Brien 1995; Myers et al 1998). In summary, we call such a problem "the issue of density-dependent catchability."

Given the historical fact, many researchers and practitioners sometimes recommend "area-wise control," which includes attempts for local extermination. Such regimes in invasive species management are that removal operations for invasive species are made only in some part of the whole habitat where some important industry or ecological asset is located such as agriculture. Real world examples in which area-wise control 
is undertaken as a management strategy include: raccoon (Procyon lotor) problems in Hokkaido, Japan, Crown-of-thorns starfish (Acanthaster planci) problems in Okinawa, Japan, and many other instances in various places. As an example from native species control problems, a sika deer management program is well-known, which has been enforced since 1998 in Hokkaido, Japan. In this program, Hokkaido is divided into several regions and area-wise control strategies are undertaken (Matsuda et al 1999).

Whereas area-wise controls have recently emerged as a scheme in invasive species management, a series of literature, which analyzes the management strategy in this vein, mainly focuses upon a situation where invasive species are reproduced and removals are implemented in a single closed system (Eisewerth and Johnson 2002; Olson and Roy 2002; Perrings 2005; Hastings et al 2006). However, such a framework is not appropriate when removal efforts are locally implemented.

It is noted by several papers that a meta-population model is more appropriate since (i) local removals potentially impact the inter- and intra-species competition, and (ii) habitat conditions may simply be heterogenous (Hastings 1982; Holt 1985; Delong and Lamberson 1999). As a result, density dependent migration may become asymmetric (Tuck and Possingham 1994; Armstrong and Skonhoft 2006). Although there may be several works which consider area-wise controls, none of them, to the best of our knowledge, explicitly examine the effect of a meta-population structure, densitydependent catchability and asymmetry in migration in the invasive species management. Thus this paper seeks to tackle these issues. At this point, several open questions come to mind:

1. What would be an appropriate measure for effectiveness of removal efforts from the long run perspective?

2. Is there any situation where it is better to aim at local extermination even with a meta-population? 
3. How do the degree of asymmetry in migration and the density-dependent catchability affect the long-run equilibrium outcome?

4. How does an economically optimal effort level change with the above two factors?

The goal and contribution of this research are to develop a simple framework of discrete-time models for analyzing the long-run consequences of removal operations for a meta-population, and to answer a set of the aforementioned questions in the context of invasive species management. In particular, an ecological model with two-patch environments is proposed, considering the key features of invasive species controls: (i) asymmetry in density dependent migration, and (ii) the effect of density-dependent catchability.

While we do not obtain analytical characteristics due to non-linearity in the form of density-dependent catchability, we demonstrate that our model could be utilized for a real world case study of invasive species management. For the purpose of illustration, the model is applied to agricultural damage control in the raccoon problem that has occurred in Hokkaido, Japan. In this application, we consider two economic functions so as to measure the effectiveness of removal effort levels: 1. agricultural damage originating from roaming raccoons and 2. removal costs which are formulated as a function of removal efforts. We first investigate the long run equilibrium outcomes of ecological variables and the associated economic functions, and then discuss an economically optimal effort level.

\section{Model}

Management officials seek to balance the cost of removal operations and damage that originates from roaming invasive species. Therefore, they are sometimes determined to implement removal operation only in some part of the whole habitat areas. This may 
be due to the fact that the whole habitat areas could be too huge to be covered by removal operation, or the budget in every period may not be sufficient to do so.

The simplest framework for the analysis of such a situation is applying an ecological model in two-patch environments (Holt 1985). The area in which removal operations are implemented is denoted as controlled patch, and the other areas in which no removal operation is implemented are denoted as uncontrolled patch.

Ecological model

Consider the following system of population dynamics over time in the two-patch environments in which removal operations are implemented only in one patch:

$$
\begin{aligned}
X_{1, t+1} & =F\left(X_{1, t}\right)-M\left(X_{1, t}, S_{2, t}\right) \\
& =r_{1} X_{1, t} /\left(1+K_{1} X_{1, t}\right)-m\left(\beta X_{1, t} / A_{1}-S_{2, t} / A_{2}\right),
\end{aligned}
$$

and

$$
\begin{gathered}
X_{2, t+1}=F\left(S_{2, t}\right)+M\left(X_{1, t}, S_{2, t}\right) \\
=r_{2} S_{2, t} /\left(1+K_{2} S_{2, t}\right)+m\left(\beta X_{1, t} / A_{1}-S_{2, t} / A_{2}\right), \\
S_{2, t}=X_{2, t}-H_{2, t}
\end{gathered}
$$

where

$X_{1, t}$ is invasive species population in the uncontrolled patch at period $t$;

$X_{2, t}$ is invasive species population in the controlled patch at the beginning of period $t$;

$r_{i}, i=1,2$ is the net growth rate of habitat $i$;

$K_{i}, i=1,2$ is the parameter of habitat $i$ related to the density feedback rate; 
$A_{i}, i=1,2$ is the area of habitat $i$

$m(>0)$ is a parameter representing the general magnitude of migration between habitats;

$\beta(>0)$ is a parameter to take account of the fact that the migration may be due to different habitat potentials within the two sub-populations caused by harvesting in controlled patch and by heterogeneous habitat conditions;

$H_{2, t}$ is population removed in the controlled patch at period $t$;

$S_{2, t}$ is escapement in the controlled patch at period $t$.

In the above model, we simply ignore heterogeneity in the density and in the migration probability within the habitat. In addition, we assume that the per capita migration rates in habitats 1 and 2 are respectively $m^{\prime} \beta A_{2}$ and $m^{\prime} A_{1}$, which is proportional to the area of destination. The numbers of migrants from habitat 1 to 2 and from 2 to 1 are respectively $m^{\prime} \beta A_{2} X_{1}$ and $m^{\prime} A_{1} S_{2}$. Replacing $m^{\prime} A_{1} A_{2}$ by $m$, we obtain Eqs. 1 and 2. Parameter $\beta$ plays a key role in determining the long run equilibrium outcomes, which represents the degree of asymmetric migration in two-patch habitats.

The above system of difference equations is similar to the one of the continuous time model employed in Armstrong and Skonhoft 2006. However, there are some distinct points to be noted. First, we choose the discrete-time setting for the purpose of applications since the time series data on raccoon population, economic damage, and estimated biological parameters are collected in the discrete manner. In fact, most basic statistics and data are yearly based in the raccoon problems. We also believe that the discrete-time formulation is more convenient for the purpose of applying the model to case studies in general.

Second, a stock-recruitment model is employed to take account of density-dependent catchability (Tuck and Possingham 1994; Clark 1990). This must be distinguished 
from a straightforward discretization of the continuous-time model as adopted in the application of fishery models. The discretization scheme as in fishery literature causes a problem that the effect of density-dependent catchability is not well-taken, whose standard specification can be found in Conrad 1999. Such a choice of discretization is more likely to yield the result that extermination is desirable. On the other hand, the stock-recruitment model enables us to incorporate the density-dependent catchability well as demonstrated in what follows.

To capture the effect of density-dependent catchability, a continuous-time submodel representing a production function is introduced in each intra-period as follows.

$$
\begin{aligned}
\dot{h}=d h / d \tau & =\left(X_{2, t}-h(\tau)\right) q\left(X_{2, t}-h(\tau)\right) e(\tau)=p\left(X_{2, t}-h(\tau)\right) e(\tau), \\
& =\left(X_{2, t}-h(\tau)\right) b\left(X_{2, t}-h(\tau)\right)^{\theta-1} e(\tau)=b\left(X_{2, t}-h(\tau)\right)^{\theta} e(\tau),
\end{aligned}
$$

where

$\tau$ denotes an instant of time in an intra-period such that $t \leq \tau \leq t+\Delta, 0<\Delta<1$, and $\Delta$ denotes the length of time in removal operation implemented in that period $t$;

$e(\tau)$ is the effort level devoted at instant $\tau$ for $t \leq \tau \leq t+\Delta$;

$h(\tau)$ is the stock size removed by operations at instant $\tau$ for $t \leq \tau \leq t+\Delta$;

$X_{2, t}-h(\tau)$ is the existing population of invasive species (escapement) at instant $\tau, t \leq$ $\tau \leq t+\Delta$

$q(\cdot)=b\left(X_{2, t}-h(\tau)\right)^{\theta-1}$ is density-dependent catchability, $b$ is some coefficient to be adjusted for measurement units and $\theta \geq 0$ is the sensitivity of catchability;

$p(\cdot)=b\left(X_{2, t}-h(\tau)\right)^{\theta}$ is catch per unit of effort (CPUE) 
with the boundary conditions that

$$
h(t)=0, \quad h(t+\Delta)=H_{2, t}, \quad t=0,1, \ldots, \infty .
$$

Combining the specifications of Eqs. 4 and 5, we analytically derive a production function of $H_{2, t}$, which is analogous to solving an initial value problem of the first-order ordinary differential Eq. 4 with the boundary conditions 5 . Solving for $H_{2, t}$ yields

$$
H_{2, t}=X_{2, t}-\left[X_{2, t}^{1-\theta}-(1-\theta) b \int_{t}^{t+\Delta} e(\tau) d \tau\right]^{\frac{1}{1-\theta}}
$$

where $E_{t}=\int_{t}^{t+\Delta} e(\tau) d \tau$ represents the total effort level of removal operations devoted by the management officials in period $t$, and the second term in the right-hand side is the escapement level in period $t$, i.e.,

$$
S_{2, t}=\left[X_{2, t}^{1-\theta}-(1-\theta) b \int_{t}^{t+\Delta} e(\tau) d \tau\right]^{\frac{1}{1-\theta}}=X_{2, t}-H_{2, t}
$$

This type of sub-continuous model in an intra-period for the production function is first introduced by Clark 1990, and many other researchers implicitly adopt such specification as well (Reed 1979; Moxnes 2003). With this approach, the effect of density-dependent catchability and CPUE that has actually occurred in each intraperiod is well-taken in the sense that the required effort level of catching one individual may rise as the existing population decreases.

For clearer understanding, refer to Fig.1 in which catchability and CPUE are graphically shown as a function of the existing population of invasive species in an intra-period. The initial population prior to any removal operation is $X_{2, t}$. As time goes on in each intra-period, removal efforts are made, and the existing population of $X_{2, t}-h(\tau)$ gradually decreases. At the same time, CPUE is monotonically decreasing, while marginal 
change in catchability depends on whether or not the sensitivity of catchability, $\theta$, is larger than unity. If it is larger than unity, catchability decreases in response to a decline in the existing population, otherwise it increases. A series of these events that occurs in each intra-period during removal operations are graphically described in Fig.1.

A parameter of our interest is the sensitivity of catchability, $\theta$, which represents the index for the percent change of catchability in response to $1 \%$ change in the existing invasive species stock. Put differently, it represents how CPUE depends on the existing population of invasive species. As Fig.1 shows, if $\theta>1$, CPUE is convex in the existing population, otherwise concave.

Here, it must be noted that if the sensitivity of catchability is larger than unity, i.e., $\theta>1$, extermination of invasive species is impossible, otherwise possible. This fact may be noticed by checking the second term in the right hand side of equation (6), that is, the escapement level is

$$
\left[X_{2, t}^{1-\theta}-(1-\theta) b \int_{t}^{t+\Delta} e(\tau) d \tau\right]^{\frac{1}{1-\theta}}=\left[X_{2, t}^{1-\theta}-(1-\theta) b E_{t}\right]^{\frac{1}{1-\theta}}
$$

This term is positive for any finite effort level of $E_{t}$ when $\theta$ is larger than unity. In other words, when $\theta>1$, the required effort level for extermination is infinite, which implies infeasibility of eradication. However, even though $\theta<1$, it does not imply that extermination is easy. In this case, as the sensitivity of catchability is approaching one, extermination gets more difficult and costly actions. The sensitivity of catchability, $\theta$, and adjustment parameter, $b$, in the production function are identified from the field data, which we describe in the calibration section.

The decision that must be made by the management officials is to set an annual effort level for removal operations, $E_{t}, t=0,1, \ldots$ In real world, this is measured by aggregate days for which traps had been set in the field. It is common that the 
government officials announce the target of total effort level they seek to achieve in each period. In this paper, it is assumed that the government sets effort levels to some constant and keeps the level all over the remaining periods, since it works as a benchmark analysis for the population dynamics.

We also admit that an optimal removal effort via dynamic programming or optimal control can be derived under the assumptions that the current estimates of population levels in two patches are accurately measured. However, we do not take this approach, and leave a topic to be addressed in the future. As is often the case with invasive species management, the population estimates especially outside the controlled patch are unavailable or not collected by the government agency. Therefore, even though it is possible to derive an optimal feedback strategy of removals, as in the sense of Tuck and Possingham 1994, it is quite difficult to be implemented due to the informational obstacles in reality. Thus, a constant annual effort is assumed along the line of the above argument, i.e., $E_{t}=E$ for all $t=\{0,1,2, \ldots\}$, but the government can choose the level of $E$.

Bionomic steady state

Under the assumption of constant effort level $E_{t}=E$, there may exist a steady state at which $X_{i, t+1}=X_{i, t}, S_{2, t+1}=S_{2, t}, H_{2, t+1}=H_{2, t}, i=1,2, t=\tilde{\tau}, \ldots, \infty$ for some $\tilde{\tau}>0$. For simplicity, we drop the subscript of $t$ to denote a set of the variables at the steady state in what follows, i.e., $X_{1}, X_{2}, S_{2}, H_{2}$. The bionomic steady state can now 
be characterized by the following system of equations:

$$
\begin{aligned}
& X_{1}\left(\frac{r_{1}}{1+K_{1} X_{1}}-1\right)=m\left(\beta X_{1} / A_{1}-S_{2} / A_{2}\right), \\
& X_{2}=r_{2} S_{2} /\left(1+K_{2} S_{2}\right)+m\left(\beta X_{1} / A_{1}-S_{2} / A_{2}\right), \\
& S_{2}=X_{2}-H_{2} \\
& \left(X_{2}-H_{2}\right)^{1-\theta}=\left(X_{2}^{1-\theta}-(1-\theta) b E\right) .
\end{aligned}
$$

The system derives from Eqs. 1, 2, 3 and 6, and possesses four unknowns of $X_{1}, X_{2}, S_{2}, H_{2}$ and four equations.

Unfortunately, it is impossible to solve the steady state equilibria in the analytic form. However, we confirm that there are two equilibria in which (i) all variables are zero, and (ii) at least some of them are interior. The stability of the latter equilibrium is checked by formulating the Jacobian matrix, following the standard procedure introduced in Edelstein-Keshet 1988. We have identified that it is stable in most plausible parameter spaces.

\section{Economic model}

We introduce two economic functions which work for measuring the effectiveness of some constant removal effort level: which are (i) costs of removal operation and (ii) social damage that accrued from roaming invasive species. While removal cost is easy to measure, what is social damage may be difficult to reach consensus. Social damage in the controlled patch could mainly be divided into the following two types; (i) agricultural economic loss and (ii) ecological one. Whereas there does not exist a good measure of ecological loss, data on agricultural economic damage has been collected by Hokkaido government, Japan (Hokkaido-government 2006). Thus, we adopt the agricultural economic loss as a proxy representing social damage. 
The operation cost for removal is taken from the standard specification of renewable resource management, i.e,

$$
C_{t}=c E_{t}
$$

where $c$ is constant marginal cost per unit effort. On the other hand, agricultural damage is assumed to be a class of the following power function of the escapement level at period $t$, i.e.,

$$
D_{t}=D\left(S_{2, t}\right)=a S_{2, t}^{d}
$$

where parameters of $a$ and $d$ are estimated from available data. We take this functional form for the two reasons. First, it is convenient for the estimation, which also can accommodate the possibility of both monotone concave and monotone convex functions simultaneously. Second, it is generally believed that the the damage function should not decrease such as bell-shaped as the escapement increases (See, e.g, Conrad 1999). Thus a power function is usually assumed for the estimation of the damage function.

Given the above economic functions of removal costs and social damage out of invasive species, we propose that the social welfare in the long run equilibrium may be a good measure of economic effectiveness from a long run perspective. As noted in the previous section, a stable equilibrium exists when government officials set some constant annual effort of $E$. In this case, it is guaranteed that all ecological variables of $\left(X_{1, t}, X_{2, t}, S_{2, t}, H_{2, t}\right)$ converges to $X_{1}, X_{2}, S_{2}, H_{2}$ in the long run, independently of the initial population levels as far as the parameters and effort level are unchanged. Of course, the steady state depends on the constant annual effort $E$, that is, the equilibrium can be reexpressed as $X_{1}(E), X_{2}(E), S_{2}(E), H_{2}(E)$. Thus, welfare in the long run equilibrium is written as

$$
W(E)=-C-D\left(S_{2}\right)=-c E-a S_{2}(E)^{d} .
$$


One of the aims in this research is to suggest an economically optimal level of constant annual effort, that is,

$$
E^{*} \in \operatorname{argmax}_{E \in \mathbb{R}_{+}} W(E)
$$

which is equivalent to finding an effort level that minimizes the social welfare loss in an interior equilibrium.

\section{Model calibration and parameter estimations}

Fig.2 displays the locations of the controlled and uncontrolled patches on raccoon management in Hokkaido, Japan. The denser colored and less colored patches in an area framed by a black line in that figure correspond to the controlled and uncontrolled patch, respectively. In this section, the model introduced in the previous section is calibrated to capture the population dynamics with density dependent migration for the purpose of application to raccoon problems in Hokkaido, Japan.

\section{Biology}

In this subsection we introduce how to determine a set of parameters necessary for the numerical analysis of population dynamics, based on the result of field research as well as the life table of raccoons in Hokkaido. We mainly focus on a net growth rate, $r$, and a parameter of $K$ related to the density dependent feedback in each patch.

With respect to the net growth rates, the governmental reports provide some benchmark method from the life table of raccoons (Hokkaido-government 2006). For this calculation of a net growth rate, several assumptions in the life table must be made: sex ratio of male and female, pregnancy rate, litter size, natural death rate and child death rates of adults and juveniles within a single year. We adopt the same values for these parameters and calculation method noted in Hokkaido report (Hokkaido-government 
2006), and finally obtain $r_{i}=1.61, i=1,2$, which is employed in a simulation throughout the rest of the paper.

With respect to the parameter of $K$, our decision is based on the recent field research conducted by Maesaki et al 2001. They report that the range of estimation in density per $\mathrm{km}^{2}$ is approximately $0.5 \sim 4.1$. Given this field survey, we adopt $4 / \mathrm{km}^{2}$ for the possible maximum density. Since we know the areas of the controlled and uncontrolled patches, which are $A_{2}=9,506 \mathrm{~km}^{2}$ and $A_{1}=38,527 \mathrm{~km}^{2}$, multiplying these with density yields the approximation of the possible maximum population level in the uncontrolled and controlled patches as 154,112 and 38,028, respectively. Given these values, we can identify $K_{i}$ by utilizing the fact that without considering density-dependent migrations, the stable steady state in each patch is derived as

$$
X_{i}=\frac{r_{i}}{K_{i}}-\frac{1}{K_{i}}>0, \quad i=1,2
$$

from Eqs. 1 and 2. Since we can consider that $X_{i}$ could be approximated by 154, 112 or $38,028, K_{1}$ and $K_{2}$ are computed as $3.958 \times 10^{-6}$ and $1.604 \times 10^{-5}$.

With respect to the remaining two parameters in the population dynamics, that is, $\beta$ and $m$, associated with migration, there is no available data or field research that can be used for identification. Instead, we suppose some range of values for these parameters, and describe how they affect the resulting outcomes in the discussion. At this point, we simply note that three values for $\beta$ are assumed $\beta=\{0.5,1.0,1.5\}$, and the rate of migration between two patches, $m=875$, as a starting point.

\section{Social damage}

A series of annual reports issued by Hokkaido government suggests that agricultural damage is a main factor that motivates her to implement removal controls of raccoons. 
Therefore, this paper takes agricultural damage or loss as a proxy for social damage as mentioned previously.

Fig.3 illustrates the relation between agricultural damage (unit: ten thousand yen) and escapement, i.e., $X_{2, t}-H_{2, t}$ collected as data over the last ten years in the controlled patch (Hokkaido-government 2006). Surprisingly enough, the curvature is not convex, but concave in the sense that marginal agricultural damage appears to be decreasing in the escapement level. This feature is opposite to the usual assumption that a series of past economic literature has adopted.

This may be due to several reasons. First, it has been remarked that the way of collecting data on agricultural damage is subject to measurement errors. For instance, farmers, who suffer from roaming raccoons in an early stage, may tend to over-report the agricultural damage due to psychological or cognitive reasons. Second, raccoons are well-known to possess opportunistic and omnivorous feeding habits (Ikeda et al 2004). They therefore may seek to obtain another source of preys if agricultural products for their forage become scarce to a certain degree. In any event, the evidence for the relation between agricultural damage and the escapement of raccoons remains scarce and the reasons must be further investigated.

We estimate the damage function of $D_{t}=D\left(S_{2, t}\right)=a S_{2, t}^{d}$ by running the following regression:

$$
\log (D)=\log (a)+d \log (e s c)+\epsilon
$$

where esc represents the escapement level of population estimated in the controlled patch as an independent variable.

The OLS regression results are reported in Table 1. As expected, escapement is not so significant even at the $10 \%$ level. However, agricultural damage seems to be dependent on the escapement level from practical consideration, and we will use these estimated values of $\hat{a}=538.30, \hat{d}=0.2169$ in numerical illustrations. 
Catchability and catch per unit of effort (CPUE)

There are not sufficient data to estimate CPUE and catchability in the controlled patch, although some data of (i) population prior to removal operations, (ii) the number of populations removed, and (iii) total effort devoted within a single year are available in specific years. For example, such data in 2006 are given as follows: Population prior to removal operation, population removed and total effort are $X_{2,2006}=4,907$, $H_{2,2006}=1,140$ and $E_{2006}=64,360$ trap days, respectively. Based on these limited data, the best thing one can do is to introduce several plausible scenarios that may be the case in reality, and identify the catchability and CPUE depending on each scenario.

The scenarios we will assume with respect to catchability and CPUE are:

1. (Eradication is Infeasible (EI)), In this scenario, we set $\theta_{E I}=1.1, b_{E I}=1.78 \times$ $10^{-6}$.

2. (Eradication is Difficult, but Feasible $(\mathrm{EDF})), \theta_{E D F}=0.9, b_{E D F}=9.48 \times 10^{-6}$.

3. (Eradication is Possible, $(\mathrm{EP})), \theta_{E P}=0.5, b_{E P}=2.69 \times 10^{-4}$.

Utilizing the above three values in 2006 and production function of equation 6 gives the parameter values of each scenario, that is, $\theta$ and $b$.

First, it must be recalled that the sensitivity of catchability, $\theta$, tells us whether extermination is feasible or not. Since we never know its true value with the current removal technology and methods for our case study, it is good to assume several possibilities. Therefore, we first set $\theta_{E I}=1.1, \theta_{E D F}=0.9, \theta_{E P}=0.5$, and each scenario is named EI, EDF, EP, respectively, after the degree of difficulty in achieving extermination corresponding to the value of the sensitivity of catchability. Once we set the sensitivity of catchability and given the values of $X_{2,2006}, H_{2,2006}, E_{2006}$ in 2006 , we can 
calculate adjustment parameter, $b$, from Eq. 6. Rearranging Eq. 6 yields

$$
b=\frac{X_{2, t}^{1-\theta}-\left(X_{2, t}-H_{2, t}\right)^{1-\theta}}{(1-\theta) E_{t}}
$$

which enables us to identify the values of $b_{E I}, b_{E D F}, b_{E P}$ as listed above.

In the result section that follows, we will compare the long run equilibrium outcomes across each scenario, which plays an important role in determining the economically optimal level of constant annual effort associated with asymmetric migration between two patches.

Result

We present numerical results obtained via Matlab in this subsection, using the parameter values and functional forms for key variables calibrated from the data in the raccoon problem, Hokkaido, Japan. Throughout this subsection, we treat the long run equilibrium outcomes under the constant annual effort. We have confirmed that all the equilibrium outcomes presented in this subsection are invariant with an initial population level. In other words, the same equilibrium outcomes, independently of initial population levels, are reached as far as the parameters and effort levels keep unchanged.

Fig. 4 provides a set of 9 panels $(3 \times 3)$, which displays equilibrium outcomes of an ecological model, depending on each scenario and parameter set. The horizonal axis in each panel represents the constant annual effort level measured by total days for which traps has been set in the field, while the vertical axis denotes ecological variables of $X_{1}, X_{2}, S_{2}$. The panels in the 1 st, 2 nd and 3 rd rows measure $X_{1}, X_{2}, S_{2}$, and the 1st, 2nd and 3rd columns correspond to the scenarios of EP, EDF and EI, respectively. Each panel provides a comparison of three lines, each of which is corresponding to the parameter of asymmetric migration: $\beta=\{0.5,1.0,1.5\}: \beta=0.5$ (line without dots), 
$\beta=1.0$ (solid line with dots), $\beta=1.5$ (thin line with dots).

As can be seen from Fig.4, the parameter of asymmetric migration, $\beta$, affects the long-run equilibrium. In general an increase in $\beta$ yields more raccoon population in the controlled patch, $X_{2}$, and less population in the uncontrolled patch $X_{1}$. This result follows our intuition.

On the other hand, difference in $S_{2}$ due to asymmetric migration of $\beta$ depends on the annual effort level of $E$. If $E$ is sufficiently small, then difference in $S_{2}$ is obvious (See the effort level of $0 \sim 300,000$ in the 3rd row panels of Fig.4). However, once $E$ gets sufficiently large, then the difference becomes small or negligible (See the effort level of 300, $000 \sim 600,000$ in the 3rd low panels of Fig.4, and also refer to Table 3). Such a trend for sufficiently large effort levels arises due to the two different reasons depending on each scenario. In EP, local extermination is simply achieved, i.e., $S_{2}=0$, for all $\beta=\{0.5,1.0,1.5\}$ when sufficiently large efforts are devoted. In fact, the effort levels required for extermination are $300,000,410,000$, and 510,000, depending on parameter values of $\beta=\{0.5,1.0,1.5\}$ (Confirm this from the row of EP in Table 3 and also from Fig.4).

In terms of EDF and EI, CPUE gets very low in an equilibrium as an effort level is sufficiently increased and the existing population in the controlled patch decreases. This feature in an equilibrium reflects the fact that effectiveness of one unit effort rapidly declines, and thus the difference in the population level prior to removal operation has negligible impacts on the resulting escapement level afterwards. Thus, difference in $S_{2}$ becomes small as effort levels are sufficiently large, although extermination is not achieved in EDF and EI (See the columns of EDF and EI in Table 3).

Here, it must be recalled that extermination is technically feasible in EDF and EP. For the range of effort levels we employed in numerical analysis, it is succeeded in EP when the effort level of $E$ are set more than 300,000, 410,000, 510,000 depending on 
$\beta=0.5,1.0,1.5$, respectively, and it is not achieved in EDF for all $\beta$ (See Table 3). This suggests that even though extermination is technically possible, the difference in the sensitivity of catchability, $\theta$, significantly affects the annual effort level at which eradication is succeeded in an equilibrium. In general, as the sensitivity of catchability is larger, the effort level that is required for extermination would increase as illustrated. In summary, analysis of an ecological model suggests that both the sensitivity of catchability and the degree of asymmetric migration are crucial in determining the equilibrium outcome especially on whether local extermination is succeeded or not in the controlled patch.

We have looked at the ecological outcomes so far. In turn, we now present the economic consequence in what follows. Fig. 5 provides a set of 9 panels $(3 \times 3)$, which displays equilibrium outcomes associated with social welfare defined in Eq. 11 where the 1st, 2nd and 3rd rows correspond to the equilibrium welfare when constant marginal cost is set as $c$ is $200,100,50$, respectively, while the 1st, 2nd and 3rd columns correspond to the scenarios of EP, EDF, and EI, respectively. Each panel provides a comparison of three lines, each of which corresponds to the parameter of asymmetric migration, $\beta=\{0.5,1.0,1.5\}: \beta=0.5$ (line without dots), $\beta=1.0$ (solid line with dots), $\beta=1.5$ (thin line with dots).

Fig.5 enables us to identify an economically optimal level of constant annual effort from the long run perspective. It is the one which gives the highest value of $W(E)$ as defined in Eq. 12. For instance, when $c=200$ and the scenario is EI, then an economically optimal effort level is zero. Because $W$ is the highest at $E=0$ (See the panel of the 1st row and 3rd column in Fig.5). It implies that any positive removal effort does not pay off compared to the case of $E=0$ for all $\beta=\{0.5,1.0,1.5\}$ in the long run. As another example, focus on the case of EI when $c=50$ (See the panel of the 3rd row and the 3rd column in Fig.5). Then it can be observed that an economically 
optimal effort level is about 220,000 for all $\beta=\{0.5,1.0,1.5\}$. This suggests that setting $E=220,000$ pays off compared to any other effort level from the long run perspective, irrespective of the degree of asymmetric migration.

Close inspection of Fig.5 reveals that the qualitative features of $W(E)$ in EP are quite different from those in EDF and EI. In EP, the economically optimal effort level is located where local extermination is just achieved if constant marginal cost is sufficiently small, i.e., $c=\{100,50\}$, otherwise zero effort is economically optimal (See the column of EP in Table 3, and the three panels of the 1st columns in Fig.5). Thus, in this situation the problem simply reduces to "Is the constant marginal cost of $c$ small enough that local extermination pays off?" When $c=\{50,100\}$, it is optimal to aim at local extermination so that an economically optimal effort levels must be adapted with $\beta$. When $c=200$, zero effort level is optimal.

Whenever the sensitivity of catchability is sufficiently small, the same qualitative feature with respect to $W(E)$ as in EP holds. In this case, economically optimal effort levels could be highly dependent upon degree of asymmetric migration, $\beta$. This reflects the fact that in $E P$, the effort level required for eradication increases as $\beta$ rises (See Table 3).

In EDF and EI, the optimal effort levels are zero or some strictly positive effort level, which could be independent of parameters of asymmetric migration $\beta$. If $c=50$, then the optimal effort level is located around 220,000 230,000 and its levels appears to be independent of the degree of asymmetric migrations $\beta$ in both scenarios (See the two panels of the 3rd row and 2nd, 3rd columns in Fig.5). Next, observe the two panels of $c=100$ (See the two panels of 2nd row and 2nd and 3rd columns in Fig.5). In EDF, only when $\beta=0.5$, it is optimal to set about $E=200,000$, otherwise zero. In EI, the optimal effort level appears to be around $E=150,000$ for all $\beta$. Finally observe the two panels of $c=200$ (See the two panels of the 3rd row, and 2nd, 3rd columns 
in Fig.5). In these cases, the optimal effort level is zero irrespective of $\beta$ so that any positive level of removal effort cannot be justified in both scenarios (See the two panels in the 1st row and 2nd, 3rd columns in Fig.5).

From a series of the above numerical results in EDF and EI, we draw the following observations: whenever the sensitivity of catchability is sufficiently high and some positive effort is economically desirable for all $\beta$, then the economically optimal effort level could be almost independent of the degree of asymmetric migration. This is in sharp contrast with the case of EP.

In this result section, we choose the limited parameter set of constant marginal cost $c=\{200,100,50\}$. However we can say what would happen if it takes other parameter ranges of $c$. If constant marginal cost of $c$ takes the value larger than 200, the optimal effort levels simply remains zero for all scenarios. If $c$ is less than 100 , the qualitative features of optimal effort levels are almost identical to the ones with the cases of $c=50$ in all scenarios. Therefore, our result presented in this section could be viewed as an exhaustive list of important results.

Finally we summarize the findings; it is demonstrated that only when the sensitivity of catchability is sufficiently small such as EP scenario, local extermination at the controlled patch yields an optimal welfare in an equilibrium. Accordingly, the optimal effort level must change with $\beta$ as illustrated above for local eradication. On the contrary, if the sensitivity of catchability is sufficiently large such as EDF or EI, it is never optimal to aim at local extermination, rather it could be better to aim at keeping low escapement level at the controlled habitat. In such a situation, an economically optimal effort level could be almost independent of the degree of asymmetric migrations $\beta$ (See the three panels of the 3rd row in Fig.5). It must be noticed that this feature is in contrast with that in the case of low sensitivity of catchability. Therefore, identifying the sensitivity of catchability in terms of current removal technology is important to 
determine a socially desirable goal as well as the relation between an economically optimal effort level and the degree of asymmetric migrations.

\section{Discussion}

The Hokkaido government currently appears to set local extermination in the controlled patch as her goal on this raccoon problem, and aims at implementing an annual effort level of $E=80,000 \sim 100,000$ trap days. From our research, the current goal is justified only if the current technology or method for removal of raccoons exhibits a sufficiently low sensitivity of catchability and the constant marginal cost per unit effort is sufficiently low. It is testable, and the estimate on the sensitivity of catchability really helps guiding where we should go on this problem.

Although we do not present all the patterns of numerical results, we confirm that migration parameter of $m$ will not qualitatively impact on the equilibrium outcomes of both ecological variables and economic functions. In other words, for the wide range of $m$, only when the sensitivity of catchability is sufficiently small, local extermination is economically desirable. However, once $\theta$ is sufficiently high, then local extermination is never optimal and keeping the low escapement by setting the optimal effort level ranged between 150,000 and 230,000. Reflecting these numerical results with real practice on the raccoon problems in Hokkaido, we recommend that effort levels be increased up to about $150,000 \sim 230,000$ if the sensitivity of catchability with current technology is sufficiently high. On the contrary, if the current removal technology possesses a sufficiently low $\theta$, the "strike level of removal effort" for local extermination must be carefully evaluated, which highly depends on the migration rate from uncontrolled areas.

In this paper, we focus on agricultural damage as a reason for raccoon controls, and demonstrate a result that it is concave in the escapement level. What if it is convex? The answer for this question is that local extermination is simply more unlikely to 
be justified in our analytic framework, since reducing the population to zero is not so an attractive option, compared to the concave damage function. Furthermore, we also have to realize that what is more important for the decision of local extermination is the degree of marginal damage from very small populations, irrespective of the curvature in damage functions. As intuitions tell us, if marginal damages from small populations are very high (low), local extermination is more (less) attractive.

This research takes the perspective that the decisions associated with "area-wise controls" in invasive species management might be legitimately analyzed through a simple deterministic meta-population model with migration. In addition, we restrict our attention to the class of "constant annual effort" as a choice for the management officials. In reality, however, the model adopted in this research could be viewed as primitive, and it is totally possible to extend it into several directions for more real policy guidance: (1) Multiple stochasticities such as growth uncertainty and implementation error could be incorporated into a model in which a Mote Carlo simulation may be of some use, (2) the optimal feedback strategy of removal controls can be derived through dynamic programming or optimal controls even under uncertainties as in the sense of Tuck and Possingham 1994, (3) the most important extension that must be made in the future is how we incorporate loss of ecological services into the analytic framework of the mathematical model.

\section{Acknowledgment}

The authors are grateful to officers of Natural Environment Division in Hokkaido Government, who supplied the materials for this work. K. Kotani and H. Ishii would like to thank G. Abe for his valuable comments and support on an early stage of this work. We also thank the members of the Global COE Program "Eco-Risk Asia" at Yokohama National University and National Institute for Environmental Studies for valuable com- 
ments and encouragement. Our gratitude goes to financial supports from the Japanese Society for the Promotion of Science through the Grants-in-Aid for JSPS Fellow (No. 1807056) and for Scientific Research C (No. 19530221). This work is also supported

by a JSPS grant to H. Matsuda. Finally, thanks are due to two anonymous referees of this journal for their comments.

\section{References}

Armstrong CW, Skonhoft A (2006) Marine reserves: A bio-economic model with asymmetric density dependent migration. Ecol Econ 57:466-476

Bomford M, O’Brien P (1995) Eradication or control for vertebrate pests. Wildl Soc Bull 23:249-255

Clark CW (1990) Mathematical bioeconomics, 2nd edn. John wiley and sons, Inc., New York

Clout MN, Veitch CR (2002) Turning the tilde of biological invasion: the potential for eradicating invasive species. In: Clout MN, Veitch CR (eds) Turning the tide: The eradication of invasive species, pp 1-3

Conrad JM (1999) Resource economics. Cambridge university press, New York

Delong AK, Lamberson RH (1999) A habitat based model for the distribution of forest interior nesting birds in a fragmented landscape. Natural resource modeling 12:129146

Edelstein-Keshet L (1988) Mathematical models in biology. McGraw Hill, Boston, Massachusetts 
Eisewerth ME, Johnson WS (2002) Managing nonindigenous invasive species: Insights from dynamic analysis. Environmental and resource economics 23:319-342

Hastings A (1982) The dynamics of a single species in a spatically varing environment: The stabilizing role of high dispersion rates. J Math Biol 16:49-55

Hastings A, Hall RJ, Taylor CM (2006) A simple approach to optimal control of invasive species. Theor Popul Biol 70:431-435

Hokkaido-government (2006) A report on plan: Racoon controls in Hokkaido. Hokkaido government, Japan, (in Japanese)

Holt R (1985) Population dynamics in two-patch environments: Some anomalous consequences of an optimal habitat distribution. Theor Popul Biol 28:181-208

Ikeda T, Asano M, Matoba Y, Abe G (2004) Present status of invasive alien raccoon and its impact in Japan. Global environmental research 8:125-131

Maesaki T, Aoyagi M, Hayashi H (2001) Feral raccoon (Procyon lotor) population and hibitats studied in Maoi and Nopporo hills near Sapporo Hokkaido. Journal of the Japanese wildlife research society 27:5-16, (in Japanese with English abstract)

Matsuda H, Kaji K, Uno H, Hirakawa H, Saitoh T (1999) A management policy for sika deer based on sex-specific hunting. Res Popul Ecol 41:139-149

Moxnes E (2003) Uncertain measurements of renewable resources: Approximations, harvesting policies and value of accuracy. Journal of environmental economics and management 45:85-108

Myers JH, Savoie A, van Randen E (1998) Eradication and pest management. Annu Rev Entomol 43:471-491 
Olson LJ, Roy S (2002) The economics of controlling a stochastic biological invasion. American journal of agricultural economics 84(5):1311-1316

Perrings C (2005) Mitigation and adaptation strategies for the control of biological invasions. Ecol Econ 52:315-325

Perrings C, Williamson M, Dalmazzone S (2000a) The economics of biological invasions. Edward Elgar, Cheltenham, UK

Perrings C, Williamson M, Dalmazzone S (2000b) Introduction. In: Perrings C, Williamson M, Dalmazzone S (eds) The economics of biological invasions, Edward Elgar, Cheltenham, UK

Reed WJ (1979) Optimal escapement levels in stochastic and deterministic harvesting models. Journal of environmental economics and management 6:350-363

Shigesada N, Kawasaki K (1997) Biological invasions: theory and practice. Oxford university press, New York

Tuck GN, Possingham H (1994) Optimal harvesting strategies for a metapopulation. Bull Math Biol 56:107-127 
Table 1: Estimates of parameters of agricultural damage

\begin{tabular}{cccc}
\hline \hline & Estimated Coefficients & Standard Errors & t stat \\
\hline $\log a$ & 6.2884 & 0.9727 & 6.4643 \\
$d$ & 0.2169 & 0.1226 & 1.7678 \\
\hline \multicolumn{4}{c}{$R^{2}=0.3424 ;$ Adjusted $R^{2}=0.2329}$.
\end{tabular}

Table 2: The baseline parameters

\begin{tabular}{ccc}
\hline \hline Parameter & Description & Value \\
\hline$\left(r_{1}, r_{2}\right)$ & Net growth rates & $(1.61,1.61)$ \\
$K_{1}$ & Parameter of density feedback rate in uncontrolled patch & $3.958 \times 10^{-6}$ \\
$K_{2}$ & Parameter of density feedback rate in controlled patch & $1.604 \times 10^{-5}$ \\
$A_{1}$ & Area in uncontrolled patch & 38,527 \\
$A_{2}$ & Area in controlled patch & 9,506 \\
$m$ & Dispersion & 875 \\
$(a, d)$ & Parameters in agricultural damage & $(538,0.2169)$ \\
\hline
\end{tabular}

Table 3: $S_{2}$ : Escapement in equilibrium

\begin{tabular}{|c|c|c|c|c|c|c|c|c|c|}
\hline & & & \multicolumn{7}{|c|}{ Constant Annual Effort, E (trap days) } \\
\hline & & & 0 & 100000 & 200000 & 300000 & 400000 & 500000 & 600000 \\
\hline \multirow{9}{*}{$\mathrm{S}_{2}$} & \multirow{3}{*}{ EP } & $\beta=0.5$ & 34196 & 21091 & 529 & 0 & 0 & 0 & 0 \\
\hline & & $\beta=1.0$ & 38028 & 26152 & 11094 & 587 & 14 & 0 & 0 \\
\hline & & $\beta=1.5$ & 41348 & 30086 & 17403 & 3253 & 372 & 4 & 0 \\
\hline & \multirow{3}{*}{ EDF } & $\beta=0.5$ & 34196 & 10651 & 1947 & 686 & 314 & 156 & 80 \\
\hline & & $\beta=1.0$ & 38028 & 15113 & 4201 & 1565 & 734 & 378 & 200 \\
\hline & & $\beta=1.5$ & 41348 & 18483 & 6292 & 2476 & 1183 & 618 & 335 \\
\hline & \multirow{3}{*}{ EI } & $\beta=0.5$ & 34196 & 7761 & 2230 & 1055 & 613 & 393 & 266 \\
\hline & & $\beta=1.0$ & 38028 & 10902 & 3696 & 1795 & 1043 & 663 & 445 \\
\hline & & $\beta=1.5$ & 41348 & 13349 & 4911 & 2422 & 1407 & 891 & 595 \\
\hline
\end{tabular}



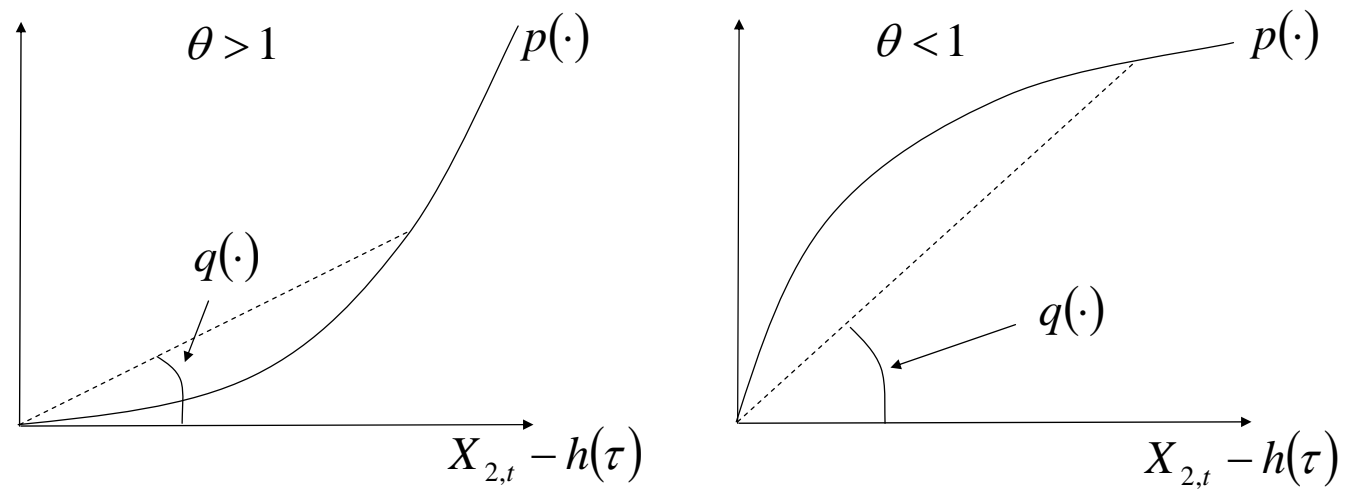

Figure 1: Catchability and CPUE as a function of the existing population size in each intra-period

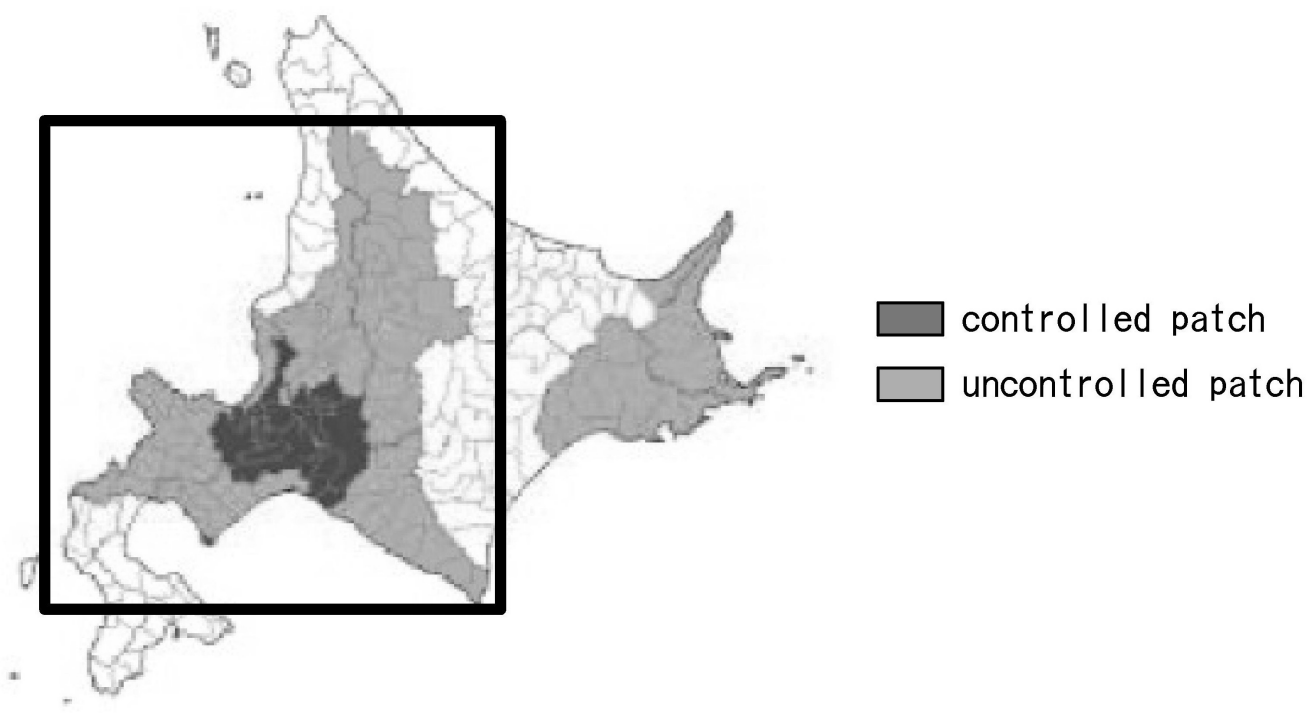

Figure 2: Location map of controlled and uncontrolled patches in Hokkaido, Japan 


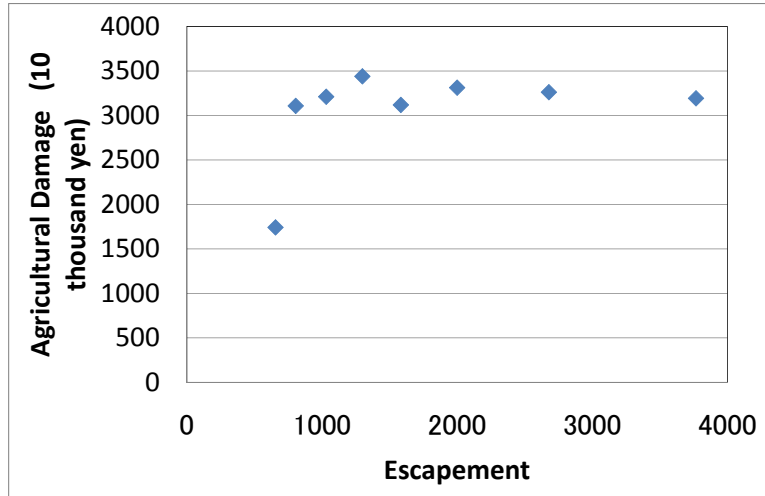

Figure 3: Scatter plot between agricultural damage and estimated escaped population 

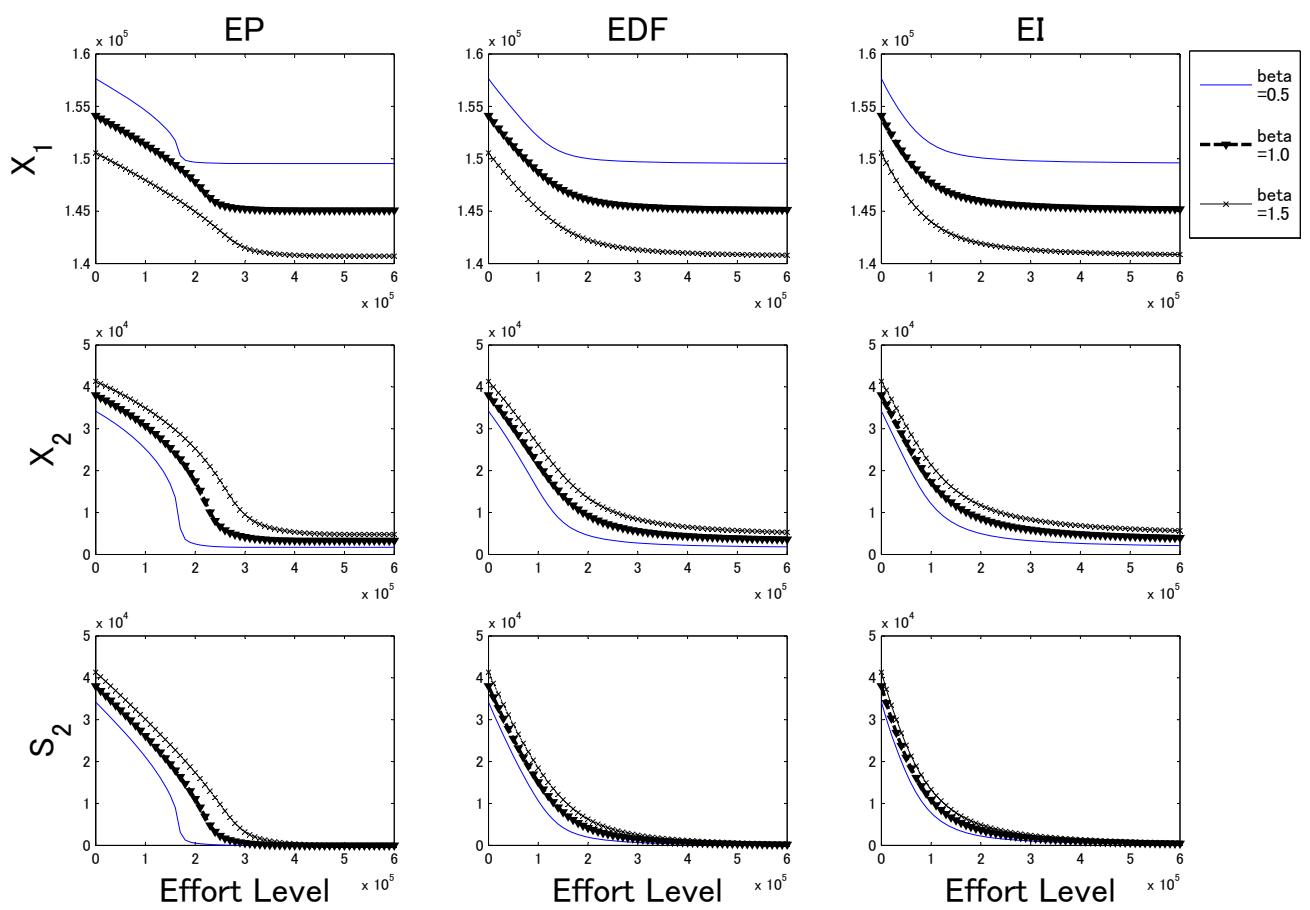

Figure 4: Equilibrium outcomes of ecological variables as a function of constant effort, $\beta=0.5$ (line without dots), $\beta=1.0$ (solid line with dots), $\beta=1.5$ (thin line with dots) where EP, EDF and EI represent the scenarios of the case that (i) eradication is possible, (ii) difficult, but feasible and (iii) infeasible, respectively. $X_{1}, X_{2}$ and $S_{2}$ are raccoon population in uncontrolled patch and the one in controlled patch, and escapement in controlled patch in the steady state, respectively. 

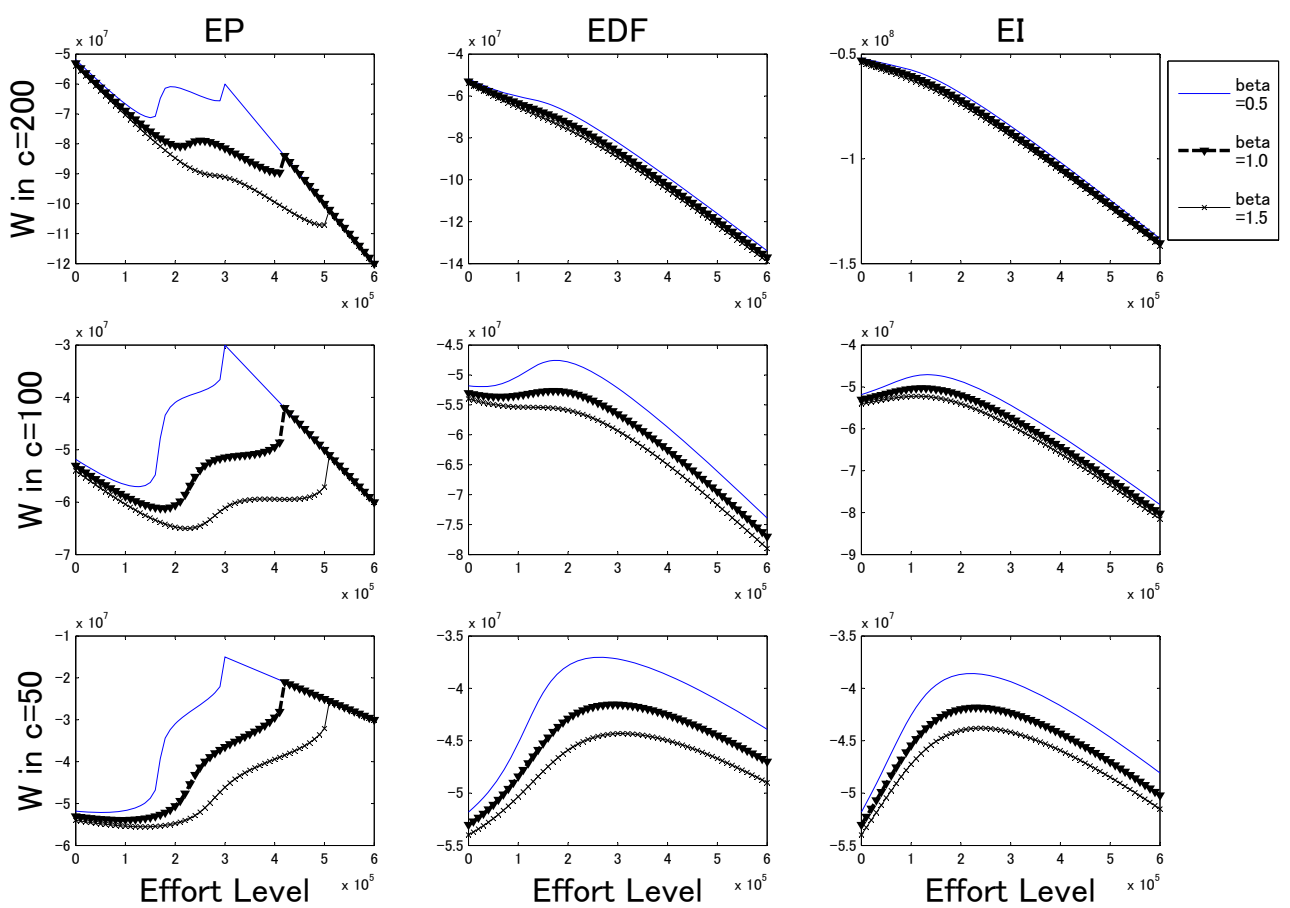

Figure 5: Equilibrium outcomes of social welfare as a function of constant effort, $\beta=0.5$ (line without dots), $\beta=1.0$ (solid line with dots), $\beta=1.5$ (thin line with dots) where EP, EDF and EI represent the scenarios of the case that (i) eradication is possible, (ii) difficult, but feasible and (iii) infeasible, respectively. $W$ and $c$ represent social welfare in a steady state and constant marginal cost per unit of removal effort, respectively. 